\title{
Food for thoughts: feeding time and hormonal secretion.
}

\author{
Celine A. Feillet
}

Short title: influence of feeding on hormone secretion profiles

Key words: circadian, feeding schedule, hormone secretion profiles

\author{
Affiliation: University of Fribourg, Department of Medicine, Division of Biochemistry, \\ Chemin du Musée 5, 1700 Fribourg, Switzerland \\ e-mail: celine.feillet@unifr.ch
}

\begin{abstract}
Many daily cycles are imposed on us by our environment, such as alternating days and nights, temperature fluctuations or rhythms in food availability. When food is accessible every day at the same time, animals will adapt their physiology and behaviour to match the daily meal. They will anticipate the access to food by waking up and being active in the hours prior to feeding, foraging for food. Adaptation of physiology to changing conditions of food availability is not only evident at the behavioural level, but also for hormonal systems. Thus corticosteroids, melatonin, leptin/ghrelin, insulin/glucagon, orexins and thyroid hormones, that show rhythmic profiles of secretion in ad libitum feeding conditions, are sensitive to increase and/or depletion in energy supplies and will be influenced when food sources are limited or available at unusual times. The purpose of the present review is to report the influence of restricted feeding schedules on secretion profiles of diverse hormones compared to normal ad libitum feeding conditions in rodents. In the end, the interplay between those systems and their response to environmental challenges will allow keeping the animal fit for survival.
\end{abstract}

This is an Accepted Article that has been peer-reviewed and approved for publication in the Journal of Neuroendocrinology, but has yet to undergo copy-editing and proof correction. Please cite this article as an "Accepted Article"; doi: 10.1111/j.1365-2826.2010.01998.x 


\section{Introduction}

All organisms living at the surface of Earth experience day/night and seasonal variations of their environment as a consequence of the Earth's rotation around the sun and around its axis. To adapt and anticipate those predictable changes, multicellular eukaryotes have almost ubiquitously developed circadian clocks (from the Latin circa: around and dies: a day; (1)) in their own way. In mammals, the suprachiasmatic nucleus of the hypothalamus (SCN) is the master circadian clock, as demonstrated both by lesion and transplantation studies $(2,3)$. The $\mathrm{SCN}$ is able to elaborate and distribute rhythmic messages of about $24 \mathrm{~h}$ to the entire organism even in complete isolation from the environment (4), thus synchronizing physiology as a whole. The $\mathrm{SCN}$ is also able to receive messages from the environment and synchronise to these messages, as long as they are in the circadian range (period between 21 and 28 hours, (5)). Among all external elements capable of synchronising the SCN, the light/dark cycle is the most powerful (photic cue). Nevertheless, in certain conditions, the SCN clockwork and its photic responses can be modulated by nutritional information (6-9). Moreover, restricted feeding schedules $(10,11)$ and other non-photic cues, like methamphetamine administration (12), have also been shown to influence behaviour and physiology outside the SCN. Thus, even if the SCN is undoubtedly the master circadian clock, other tissues around the body will be influenced, directly or not, by the environment. Consequently, many physiological parameters such as hormones will see the phase and/or amplitude of their rhythm influenced by a change in food timing and quantity. The aim of this review is to report the effect of restricted feeding on hormonal regulation in rodents. After a quick overview of the impact of restricted feeding on behaviour, we will explain in more details how conditions of limited food access can impact on hormone secretion.

\section{Influence of feeding on behaviour}

In 1979, F. K. Stephan and colleagues showed that in rats made arrhythmic by 
bilateral lesions of the $\mathrm{SCN}$, a circadian bout of locomotor activity can be restored when the animals are submitted to restricted feeding (RF). In this study, the authors allowed an access to food limited to a few hours a day without calorie restriction, delivered every day at the same time (temporal restriction). Other studies used a limited amount of food (calorie restriction) given everyday at the same time $(6,13)$. Both protocols are considered as RF. In these conditions, the animals start to be active in the hours prior to food access, thus restoring circadian locomotor activity (so called the Food Anticipatory Activity: FAA; (14) see Fig. 1). This phenomenon is not limited to wheel-running activity but is also observed in general cage activity, food bin approach or unreinforced lever pressing (15). Core body temperature also rises in the hours preceding food access, defining a Food Anticipatory Thermogenesis (FAT) (15-17). In addition, it has been established that the FAA is expressed in SCN-intact animals housed in constant darkness (DD, e.g. (15)), or in LD when food is presented during the inactive phase $(6,7)$. Moreover, food anticipation is not only present in laboratory conditions, but also in nature: doe rabbits nurse their pups only once a day, everyday at the same time. Pups are only allowed a brief bout of suckling ( $<5 \mathrm{~min}$ a day). They have to ingest up to $35 \%$ of their body weight in milk. In response to that natural restricted feeding schedule, pups show increased activity, temperature and corticosterone in anticipation to the daily meal (18). This indicates FAA is expressed early in life and is not observable in laboratory conditions only.

It is worth mentioning that FAA and FAT are currently thought to be outputs of a circadian clock distinct from the SCN, The Food Entrainable Clock, the location and functioning of which remain unclear. Then locomotor activity and temperature will be indirectly influenced by food availability via the Food Entrainable Clock. Considering current controversial knowledge on the Food Entrainable Clock and the lack of knowledge on possible direct control of hormonal secretion by this clock, I will consider in the following sections, the effect of food timing on hormone secretion, regardless of the fact that it is direct or indirect 
via the control of a clock.

\section{Corticosteroids}

In case of a stress, the paraventricular nucleus of the hypothalamus (PVN) integrates informations from limbic and brain stem centers to answer both psychological and physical stressors. In this structure, Corticotropin-Releasing Hormone (CRH) and Arginine VasoPressin (AVP) rapidly increase; in the end they activate the corticotroph cells of the anterior pituitary. Adreno-CorticoTropin Hormone (ACTH) is then released into the circulation, targeting the adrenal cortex which secretes glucocorticoid hormones (19). They will act on their receptors to trigger various responses (e.g. on the liver for energy mobilization, on immune organs to suppress innate immunity (20).

Apart from this acute phenomenon, a rhythm in glucocorticoid levels is controlled by interplay of two systems:

1- The circadian system, through GABAergic projections from the SCN to the PVN, inhibits neuronal activity in that area during the day. During the night, autonomous neurons in the PVN become active again, both because of an activation from SCN glutamatergic neurons and a suppression of GABAergic inhibition from the SCN (21). Thus the SCN stimulates CRH release from the PVN. CRH then goes into the hypothalamo-hypophyseal portal system and triggers synthesis of ACTH by the anterior pituitary. As for a stress response, secretion of ACTH in the general circulation stimulates in the end synthesis and release of glucocorticoids by the adrenal cortex. Depending on the species, steroidogenesis gives rise to liberation of a main glucocorticoid: corticosterone (rat), cortisol (human and Syrian hamster) or a combination of both (Djungarian hamster). Note that apart from the main glucocorticoid, traces of other glucocorticoids may still be detected.

2- In addition to this humoral control of glucocorticoid secretion via $\mathrm{CRH}$ and ACTH, the sympathetic system exerts a nervous control over the adrenal gland, modulating its sensitivity 
to ACTH on a daily basis (22).

In rats and mice under RF, like for temperature or locomotor activity, a peak of corticosterone appears in anticipation to food access (23-25). This peak adds to the one expressed at the end of the day/beginning of the night (just before the active phase). Corticosterone levels lower rapidly when food is provided again (Fig. 2a). Also, when water access is limited to $30 \mathrm{~min}$ during daytime, which concomitantly reduces food access, thus mimicking RF, the same anticipatory peak in corticosterone secretion is observed, but only for a chronic treatment. An acute treatment does not have the same effect (26). Corticosterone being usually associated with stress conditions, the possibility that RF was a stress was raised, with would explain the daily peak of corticosterone. However, Wilkinson and collaborators demonstrated that the anticipatory peak of corticosterone secretion does not correlate with an ACTH peak at the same time while stress response always triggers ACTH secretion (27). This early experiment was recently confirmed by Girotti and colleagues who explored the influence of RF at different levels of the hypothalamo-pituitary axis (HPA). Hence, during daytime RF, a peak of corticosterone appears in anticipation to mealtime, but ACTH levels seem not to be affected (28). It is thus possible that sensitivity of the adrenal to ACTH is increased in anticipation to mealtime while ACTH levels remain unchanged compared to ad libitum fed animals. The authors also noticed an inversion of $c r h$ patterns in the PVN, peaking during the night under RF instead of the day for ad libitum fed animals. Another group found that corticosterone anticipatory peak is not accompanied by an increase in $c r h m R N A$ in the PVN (29). This result is compatible with the preceding one as Girotti and collaborators report and increase in $c r h$ mRNA 12 hours away from the anticipatory peak of corticosterone.

As "stress hormones" corticosteroids will promote alertness in animals in anticipation to mealtime. This will correlate with the anticipatory activity and prepare for food seeking. Also, considering the wide distribution of glucocorticoid receptors, and the known impact of glucocorticoids on cell synchronisation (30), a shift in the pattern of glucocorticoid secretion 
or a second peak appearing in anticipation of food access during RF, will impact on general physiology in the animals; in a way it could inform other organs that it is time to be awake and forage for food. But of course, it will not be the only signal as will be shown in the next sections.

\section{Melatonin}

Rhythmic synthesis of the hormone melatonin by the pineal gland occurs only at night (Fig. $2 \mathrm{~b} ;(31))$ and many studies have demonstrated that it is tightly controlled by the SCN, through a now well-known polysynaptic pathway. Briefly, The $\mathrm{SCN}$ sends projections to the Paraventricular Nuclei of the Hypothalamus (PVN) which then contacts the Inter-MedioLateral column of the spinal cord (IML). The next relay is the superior cervical ganglions (SCG) projecting in the end to the pineal gland $(32,33)$. In the pineal gland, the rate limiting enzyme for melatonin synthesis is the Aryl-alkylamine N-acetyl transferase (AA-NAT). Both the aa-nat mRNA and melatonin release rhythms are abolished by a lesion of any element of the polysynaptic pathway, thus confirming their implication in melatonin rhythmicity (34-36). Melatonin secretion has been shown to be sensitive to restricted feeding and particularly to calorie restriction that advances its phase by 3 hours, but it seems that this effect is exerted via the SCN (Fig. 2b; $(6,37)$ ). Until recently, it was thought that melatonin synthesis was an exclusive output of the SCN. We demonstrated that a daily RF can impact on pineal melatonin synthesis in SCN-lesioned rats that were behaviourally arrhythmic (38). In the pineal gland of these animals, c-FOS expression, aa-nat mRNA and melatonin secretion were arrhythmic. RF restored not only a rhythmic transcription of c-FOS and a rhythmic expression of aa-nat, but also a rhythmic synthesis of melatonin in the pineal gland (38). Melatonin then peaks about 14 hours after food has been provided. To better understand how the pineal gland could be influenced by feeding cues in the absence of a functional SCN, we then tested possible indirect ways for feeding conditions to reach the pineal gland. RF triggers not only a 
peak of locomotor activity, but also a peak of corticosterone secretion in anticipation to mealtime that could both feedback on the pineal. Indeed corticosterone can potentiate noradrenergic stimulation of aa-nat transcription $(39,40)$ while locomotor activity can feedback on the sympathetic nervous system $(41,42)$. Testing those hypotheses, we came to the conclusion that the effect of RF cannot be mimicked by a daily peak of corticosterone (as induced by a daily stress in our experiment) and that a daily bout of locomotor activity is required to fully restore melatonin synthesis in the pineal gland of SCN-lesioned rats (38). It is also possible that daily synchronizing cues associated with RF are capable of activating cerebral regions at some levels of the multi-synaptic pathway downstream of the SCN to the pineal gland (i.e. PVN, IML or SCG), thus triggering in the end melatonin secretion by the pineal gland. Note that the PVN have already been demonstrated to react to RF $(43,44)$ and would be a target of choice to investigate this question.

One might ask why it could be important for the pineal gland to be sensitive to feeding conditions? One possible answer is: to communicate. Melatonin receptors have been found in multiple brain structures and peripheral tissues, in both mammalian and non mammalian vertebrates (see (31) and references therein). When the SCN is not functional, many synchronising cues will disappear and, in the absence of a master clock, there is a risk of desynchronisation between organs, resulting in inadequate timing of a myriad of physiological processes. This feature has been exemplified by Yoo and colleagues who observed circadian rhythms in tissue explants from arrhythmic SCN-lesioned mice. Each tissue did not lose circadian rhythms, but instead became desynchronized from other tissues within the same animal (45). In this context, if the pineal gland (or an upstream structure) is sensitive to recurring feeding events, it will send a daily signal via melatonin secretion to all organs presenting melatonin receptors, thus synchronizing overt physiology again. In this context, a sensitivity of the pineal gland to feeding reaches full significance. Of course what is true for the pineal gland and melatonin will also apply to all hormones cited in this review. 


\section{Leptin and ghrelin}

Leptin and ghrelin are peptides released by white adipose tissue and oxyntic cells in the stomach, respectively. They carry humoral signals from the periphery to neuronal networks regulating energy homeostasis. Neuropeptide Y (NPY) neurons in the arcuate nucleus of the hypothalamus (ARC) are their main targets. It is known that NPY is a potent appetite stimulating peptide. Ghrelin and leptin respectively stimulate and inhibit NPY expression and release, thus increasing or lowering food intake. To that respect, ghrelin has been demonstrated to reverse leptin effect on food intake when infused centrally (46). In the end leptin stimulates energy expenditure while ghrelin promotes lipid storage in the adipose tissue ((47) for review).

Leptin and ghrelin are both rhythmic in the plasma in ad libitum feeding conditions (SCN intact animals), peaking during the night for leptin (i.e. when nocturnal rodents like rats and mice normally feed) and during the day for ghrelin (Fig. 2c-d). These rhythms are inverted in response to a daytime RF, leptin and ghrelin being maximal in the plasma after and before the meal respectively (Fig. 2c-d; $(48,49)$, showing that those two hormones are sensitive to food availability. Additionally, FAA is reduced in mice knock out for ghrelin receptors (50) indicating that ghrelin is not only sensitive to feeding conditions but also participates in synchronisation to feeding schedules. Moreover, a calorie restriction (50\% of normal intake for 12 days) in rats, as well as a low fat diet in mice lead to a decrease in leptin release (51, 52). This mechanism may ensure that feeding will be triggered as soon as plenty of food is available again, then preventing critical lowering of energy stores.

\section{Orexins}

In 1998, two groups simultaneously identified two closely related peptides as endogenous ligands for two orphan receptors, named "orexins" by one group and "hypocretins" by the 
other, that proved to be the same molecule. Orexin-A and B (also called Hypocretin-1 and 2) are small neuropeptides processed from a common precursor, preproorexin. Their receptors (orexin-1 and orexin-2 receptors) are found mainly in the forebrain, including hypothalamic and thalamic regions, as well as in the brainstem nuclei and the spinal cord $(53,54)$.

Orexins were initially identified as regulators of feeding behaviour, firstly because they are produced exclusively in the lateral hypothalamic area, a region involved in the regulation of feeding, and secondly owing to their pharmacological activity: central injection of orexins during the light period stimulates food intake (55). Subsequently, the finding that a lack of orexins or a deficiency in its receptor causes a pathology mimicking narcolepsy in animals, indicated that these hypothalamic neuropeptides also play a crucial role in regulating sleep and wakefulness. Narcolepsy is a neurological disorder characterised by excessive daytime sleepiness, disrupted nocturnal sleep, rapid eye movement (REM) sleep occurring at the onset of sleep and cataplexy (a sudden loss of skeletal muscle tone) in response to strong emotional stimuli such as laughing (see $(56,57)$ for review).

Considering the importance of orexins in the coordination of wakefulness and motivated behaviours like food seeking, Akiyama and colleagues tested activation of orexin neurons (cFOS immunoreactivity) under daytime RF, and FAA in wild type and orexinergic neuronablated mice. Orexinergic neuronal activation is advanced by $6 \mathrm{~h}$ in wild type mice in response to daytime RF showing that orexinergic system can be influenced by food timing (Fig. 2g). Interestingly, FAA is reduced in orexinergic neuron-ablated mice under the same conditions. It seems that orexinergic neurons would participate in the wakefulness component of FAA during RF (58). These results were confirmed by another group that studied the involvement of orexin in the establishment and maintenance of FAA induced by RF (59). As already mentioned for corticosteroids, a system like orexins that promotes wakefulness will be of primary importance when food supplies are low: it will incite animals to be awake at unusual time of the day and seek for food, which may in turn increase survival rate as the 
animals will be able to search for food in new temporal niches.

\section{Insulin and glucagon}

Insulin and glucagon are endocrine peptides secreted by beta- and alpha-cells respectively in pancreatic islets that are responsible for glucose homeostasis. Insulin causes hypoglycaemia, meaning that it favours glucose transport and entry in skeletal muscles and adipose tissue, and stimulates glycogen synthesis in the liver, thus promoting an anabolic state. On the other hand, glucagon induces a catabolic state, with a hyperglycaemia as a consequence $(60,61)$. Both insulin and glucagon dynamically respond to food intake: after a meal, blood glucose concentration is increased; in response to this hyperglycaemia, insulin secretion is increased and glucagon secretion is reduced. Conversely, a hypoglycaemia will stimulate glucagon secretion and inhibit insulin release, ultimately raising blood glucose levels $(60,61)$. It is noteworthy that the pathology diabetes mellitus, where chronic hyperglycaemia occurs, is a consequence of either an insufficient insulin secretion (type 1) or of an insulin resistance leading to a lack of insulin action in peripheral tissues (type 2). Also, an excessive secretion of glucagon can contribute to hyperglycaemia in diabetes (62).

In ad libitum feeding conditions, insulin is minimal before day/night transition i.e. before nocturnal rodents become active and usually eat (Fig. 2e). At that time, glucagon is maximal, indicating a dominant catabolic state (Fig. 2f; $(63,64)$. As sensors and regulators of blood glucose levels, insulin and glucagon will respond to a RF. When food is restricted to daytime, insulin will reach its nadir in the hours before food access and glucagon levels will be high. After food has been provided, this tendency is inverted: plasma insulin levels become higher and glucagon levels diminish (Fig. 2e-f). The animal has returned to an anabolic state (64). This response is not limited to a unique meal. Indeed, insulin will respond whenever food is presented. Thus it has been demonstrated that rats fed six 10 minutes-meals a day, meals being separated by 4 hours, each meal is followed by an increase in insulin release in blood 
circulation (63). Glucagon has not been tested in this protocol, but it is probable that both hormones dynamically react to increase and depletion in glucose no matter when it happens. This will guarantee glucose homeostasis; considering that glucose is the exclusive source of energy for neurons, its availability and regulation are crucial for the animal.

\section{Thyroid hormones}

The thyroid hormones $(\mathrm{TH}), \mathrm{T} 3$ and $\mathrm{T} 4$, play roles in numerous physiological processes, from Development and growth to homeostasis and cellular metabolism. In the hypothalamus, PVN liberate TSH-releasing hormone (TRH) which stimulates secretion of TSH (thyroidstimulating hormone, or thyrotropin) from the anterior pituitary. TSH then triggers $\mathrm{TH}$ synthesis in the thyroid gland. The thyroid gland releases a combination of T4 and T3, produced in lower amounts, but which is the biologically active form. Serum concentrations of $\mathrm{TH}$ are tightly controlled by a negative feedback, where $\mathrm{TH}$ regulate their own synthesis by inhibiting TRH and TSH transcription directly inside the hypothalamus and the pituitary, through $\mathrm{TH}$ receptors $(65,66)$. In addition to TH receptors, a local control of TH activity will be exerted by cellular deiodinase enzyme systems, that will convert T4 to T3 locally as distribution of deiodinases varies between tissues and each one has a distinct developmental profile (67).

Regarding daily profiles of secretion, both T4 and T3 levels show nycthemeral variations with maximum levels around the middle of the day, when nocturnal animals are asleep, and a nadir at the beginning of the night phase (Fig. 2h). Like other anterior pituitary hormones, TSH shows a clear rhythm in both male and female rats, with inverted patterns compared to T4 and T3 $(68,69)$.

As previously mentioned, TH widely influence physiology. During development for example, TH deficiency or excess can impair cell differentiation and migration. Some studies suggest that T3 would influence oligodendrocyte and neuron differentiation (70). Later, tightly controlled levels of circulating thyroid hormones help maintain normal pituitary Growth 
Hormone (GH) secretion (71). In addition to those features, a link between TH and nutritional signals has been observed that is of primary interest for the present review: enhanced glucose production, absorption and utilization are associated with hyperthyroidism, whereas diminished glucose utilization and lipogenesis correlates with hypothyroidism (72).

TH have an effect on glucose metabolism and the influence of dietary restrictions on thyroid physiology has also been investigated widely. When female rats are repeatedly calorie restricted early in life (21 days of age), growth is retarded during those periods and reinitiated when food is returned to normal. Additionally, blood levels of TSH, T4 and T3 are lowered by this treatment showing that dietary restrictions decreases thyroid function (Fig. 2h). Unfortunately, this study did not explore the influence of this diet on TH rhythms, but suggests early influence of feeding on thyroid function (73). Nevertheless, it seems that a restriction of water access to 30 min during daytime, that is accompanied by a reduction in food intake does not shift patterns of circadian TSH rhythms, neither for acute nor for chronic treatment $(26,74)$. This study confirms that water/food restriction lowers TSH levels even if it does not resynchronise its rhythm. If food only is restricted to $50 \%$ of normal intake distributed in one meal, TSH, T4 and T3 remain rhythmic while T3 rhythm is lost if the same quantity of food is distributed in 5 distinct meals (74). Also note that the water/food type of restriction has the same effect on $\mathrm{GH}$, that is physiologically linked to $\mathrm{TH}$, with a lower secretion but no effect on its circadian pattern (26). Moreover, the effect of RF on TSH and GH seems to be independent of a feedback from the thyroid gland as it is also observed in thyroidectomised rats $(75,76)$. In the end, it seems food will highly impact on the Hypothalamo-Pituitary-Thyroid axis, but more than the feeding pattern, the amount of food available will be the critical parameter. When considering the links between $\mathrm{TH}$ and $\mathrm{GH}$, it is a great advantage to limit growth in cases of unfavourable energy balance and promote growth when food is available. 


\section{Crossroads}

All systems investigated with respect to RF have been shown to react their own way to adapt to challenging food availability. But all those systems are not isolated from one another and many studies have found crosstalk between different systems. For instance, at the molecular level, glucocorticoid responsive elements situated in the promoter of genes such as AA-NAT, the rate limiting enzyme in melatonin synthesis, can activate their transcription, thus linking those two systems. Also, melatonin secretion at night is decreased in stressed or glucocorticoid-treated rats, which once again shows a link between those systems $(77,78)$.

At the cellular level, added to the wide presence of glucocorticoid receptors and melatonin receptors, other precited systems can influence one another through cellular receptors; for instance, orexin producing cells have receptors for leptin and receive inputs from arcuate NPY neurons (79).

Melatonin itself will influence other systems, such as the thyroid gland, as reported in the 1980s where experiments on rats and hamsters showed that the pineal gland had an inhibitory action on the thyroid axis (80). Also, recent data suggest that exogenous melatonin, when given intraperitoneally, acts as a modulator of enzyme secretion in the pancreas, and more precisely on amylase secretion (81).

Linking the leptin/ghrelin and insulin/glucagon system, a correlation has been found between plasma ghrelin levels and elevated fasting insulin levels and insulin resistance. Moreover, ghrelin inhibits insulin release in mice, rats and humans (82).

All those features are only a drop in a sea of intertwined mechanisms. They pinpoint the complexity of interactions that take place in normal feeding conditions that will be of primary importance when food is scarce to synchronise all force towards survival. But why would a "synchronized" animal be fitter for survival? It has already been mentioned that in arrhythmic $\mathrm{SCN}$-lesioned mice, peripheral tissues continue to tick with their own phase, different from one another (45). Internal desynchrony between physiological functions can happen not only 
when the SCN is lesioned, but also following jet-lag or shift work $(83,84)$. When desynchronisation persists, it will increase mortality in aged animals (83). So it seems that having all systems ticking in accordance with each other confers an advantage for survival although the exact cause remains unknown. Whether food anticipation itself has an adaptative significance is also supposed but has never been demonstrated. The fact that it developed in many species from insects to mammals (85) argues for its relevance as it does for circadian rhythms in general $(85,86)$.

\section{Conclusion and perspectives}

Light is the main synchronizer for the biological clock residing in the SCN. But it is now unavoidable to count with another synchronizer, yet often absent from circadian biology manuals: food. If it is essential to live according to the light/dark cycle, it is even of greater importance for survival to seek for enough food. In the absence of a functional master clock, systems like melatonin or glucocorticoids that will be synchronised by feeding cues and have widely distributed receptors should be able to relay the food timing information to the whole body to synchronise physiology. Systems promoting wakefulness and alertness, such as orexins and glucocorticoids, will have to be active, even at unusual times (e.g. during the day for nocturnal animals) to allow animals to forage for food in case of energy shortage. And when food is found, it is of use to be able to process nutrients, promote storage for further utilisation when energy supplies will be low again. This is where glucose homeostasis hormones will enter into action (Insulin, glucagon). Also, triggering growth mechanisms while in a low energy context could result in damages to the integrity of the animal and that may be one reason why thyroid function should be lowered in case of energy restriction. In the end, all those systems, receiving information on energy can communicate with one another to optimise metabolism, adapting physiology to environmental conditions. In this context, it is easy to understand why any alteration in any of those systems, following 
deregulation or disease, will have great impacts on whole body integrity, and not only at the metabolic level.

One weak point remains in that highly organised structure: how do those hormonal systems synchronise to food? What are the mechanisms underlying reorganization of the entire physiology of the animal according to food timing?

One possible hypothesis would be that the Food Entrainable Clock, that is thought to control food anticipatory physiology and behaviour, is reset by food - like the $\mathrm{SCN}$ is reset by light. In turn, it will send projections to various areas of the brain (e.g. PVN, pineal gland, lateral hypothalamic area) to control and synchronise all cited hormonal systems. Once we will have access to the anatomical localisation of this clock, tracing studies can be performed to map possible nervous output pathways and neurotransmitter systems. Unfortunately, the current controversies on this clock's location (87) and clockwork (88) renders impossible such an approach at the moment.

Another possibility is that each system can be independently synchronised by food timing, responding directly to fluctuations in concentration of glucose and/or other metabolites. For example, the insulin/glucagon system seems to "passively" respond to glucose without obvious participation of any clock or synchronising mechanism (63). Moreover, it is known that some neurons in the brain exhibit specific excitatory and inhibitory electrical response to glucose (e.g. Orexin neurons) (89). These neurons, specifically linked to a hormonal system, may be the starting point of a broader food synchronisation. If they express clock genes, they can also be food entrainable oscillators or clocks.

In between those two hypotheses, lies the possibility that one system will be directly sensitive to feeding conditions and will synchronise all the other systems by means of receptors or regulatory elements. Glucocorticoids are particularly interesting to that respect as they respond to RF, can synchronize cells in culture (30) and can regulate many genes by means of 
glucocorticoid responsive elements in their promoter, as it is the case for the aa-nat gene (39, 40).

But then, how to unravel those mechanisms? If one would like to investigate the genetic basis for food entrainment in a given system, they could use microarrays in rodents under $a d$ libitum versus restricted feeding at the very beginning of RF to check for "food-entrainable early genes" (90). A genome-wide promoter analysis would also reveal which systems are susceptible to influence one another. Tracing studies from a putative Food Entrainable Clock would reveal a nervous control of a particular hormonal system. However, these approaches seldom give strait forward results. It is likely that new techniques and new concepts will be needed to fully understand the ins and outs of hormonal system entrainment by feeding.

\section{Acknowledgments}

I am indebted to Dr. Etienne Challet and Pr. Dr. Urs Albrecht for correcting this manuscript and providing helpful comments.

\section{References}

1. Dunlap JC. Molecular bases for circadian clocks. Cell. 1999; 96(2): 271-90.

2. Moore RY, Eichler VB. Loss of a circadian adrenal corticosterone rhythm following suprachiasmatic lesions in the rat. Brain Res. 1972; 42(1): 201-6.

3. Ralph MR, Foster RG, Davis FC, Menaker M. Transplanted suprachiasmatic nucleus determines circadian period. Science. 1990; 247(4945): 975-8.

4. van Esseveldt KE, Lehman MN, Boer GJ. The suprachiasmatic nucleus and the circadian time-keeping system revisited. Brain Res Brain Res Rev. 2000; 33(1): 34-77.

5. Daan S, Aschoff J. the entrainment of circadian systems in handbook of behavioural neurobiology. In: Takahashi JS, Turek FW, Moore RY, eds. handbook of behavioural neurobiology. New York: kluwer academic / plenum publishers 2001.

6. Mendoza J, Graff C, Dardente H, Pevet P, Challet E. Feeding cues alter clock gene oscillations and photic responses in the suprachiasmatic nuclei of mice exposed to a light/dark cycle. J Neurosci. 2005; 25(6): 1514-22.

7. Castillo MR, Hochstetler KJ, Tavernier RJ, Jr., Greene DM, Bult-Ito A. Entrainment of the master circadian clock by scheduled feeding. Am J Physiol Regul Integr Comp Physiol. 2004; 287(3): R551-5.

8. Challet E, Caldelas I, Graff C, Pevet P. Synchronization of the molecular clockwork by light- and food-related cues in mammals. Biol Chem. 2003; 384(5): 711-9. 
9. Lamont EW, Diaz LR, Barry-Shaw J, Stewart J, Amir S. Daily restricted feeding rescues a rhythm of period2 expression in the arrhythmic suprachiasmatic nucleus. Neuroscience. 2005; 132(2): 245-8.

10. Mendoza J. Circadian clocks: setting time by food. J Neuroendocrinol. 2007; 19(2): $127-37$.

11. Waddington Lamont E, Harbour VL, Barry-Shaw J, Renteria Diaz L, Robinson B, Stewart J, Amir S. Restricted access to food, but not sucrose, saccharine, or salt, synchronizes the expression of Period2 protein in the limbic forebrain. Neuroscience. 2007; 144(2): 402-11. 12. Iijima M, Nikaido T, Akiyama M, Moriya T, Shibata S. Methamphetamine-induced, suprachiasmatic nucleus-independent circadian rhythms of activity and mPer gene expression in the striatum of the mouse. Eur J Neurosci. 2002; 16(5): 921-9.

13. Challet E, Malan A, Pevet P. Daily hypocaloric feeding entrains circadian rhythms of wheel-running and body temperature in rats kept in constant darkness. Neurosci Lett. 1996; 211(1): 1-4.

14. Stephan FK, Swann JM, Sisk CL. Entrainment of circadian rhythms by feeding schedules in rats with suprachiasmatic lesions. Behav Neural Biol. 1979; 25(4): 545-54.

15. Mistlberger RE. Circadian food-anticipatory activity: formal models and physiological mechanisms. Neurosci Biobehav Rev. 1994; 18(2): 171-95.

16. Challet E, Pevet P, Vivien-Roels B, Malan A. Phase-advanced daily rhythms of melatonin, body temperature, and locomotor activity in food-restricted rats fed during daytime. J Biol Rhythms. 1997; 12(1): 65-79.

17. Feillet CA, Ripperger JA, Magnone MC, Dulloo A, Albrecht U, Challet E. Lack of food anticipation in Per2 mutant mice. Curr Biol. 2006; 16(20): 2016-22.

18. Caba M, Gonzalez-Mariscal G. The rabbit pup, a natural model of nursing-anticipatory activity. European Journal of Neuroscience. 2009; 30(9): 1697-706.

19. Lightman SL. The neuroendocrinology of stress: a never ending story. $J$ Neuroendocrinol. 2008; 20(6): 880-4.

20. Herman JP, Figueiredo H, Mueller NK, Ulrich-Lai Y, Ostrander MM, Choi DC, Cullinan WE. Central mechanisms of stress integration: hierarchical circuitry controlling hypothalamo-pituitary-adrenocortical responsiveness. Front Neuroendocrinol. 2003; 24(3): 151-80.

21. Perreau-Lenz S, Pevet P, Buijs RM, Kalsbeek A. The biological clock: the bodyguard of temporal homeostasis. Chronobiol Int. 2004; 21(1): 1-25.

22. Kalsbeek A, Buijs RM. Output pathways of the mammalian suprachiasmatic nucleus: coding circadian time by transmitter selection and specific targeting. Cell Tissue Res. 2002; 309(1): 109-18.

23. Krieger DT. New studies on the experimental alteration of the circadian periodicity of plasma corticosteroid levels in the rat. Chronobiologia. 1974; 1 Suppl 182-90.

24. Nelson W, Scheving L, Halberg F. Circadian rhythms in mice fed a single daily meal at different stages of lighting regimen. $J$ Nutr. 1975; 105(2): 171-84.

25. Honma KI, Honma S, Hiroshige T. Feeding-associated corticosterone peak in rats under various feeding cycles. Am J Physiol. 1984; 246(5 Pt 2): R721-6.

26. Armario A, Jolin T. Effects of water restriction on circadian rhythms of corticosterone, growth hormone and thyroid stimulating hormone in adult male rats. Physiol Behav. 1986; 38(3): $327-30$.

27. Wilkinson CW, Shinsako J, Dallman MF. Daily rhythms in adrenal responsiveness to adrenocorticotropin are determined primarily by the time of feeding in the rat. Endocrinology. 1979; 104(2): 350-9. 
28. Girotti M, Weinberg MS, Spencer RL. Diurnal expression of functional and clockrelated genes throughout the rat HPA axis: system-wide shifts in response to a restricted feeding schedule. Am J Physiol Endocrinol Metab. 2009; 296(4): E888-97.

29. Poulin AM, Timofeeva E. The dynamics of neuronal activation during food anticipation and feeding in the brain of food-entrained rats. Brain Res. 2008; 1227128-41.

30. Balsalobre A, Brown SA, Marcacci L, Tronche F, Kellendonk C, Reichardt HM, Schutz G, Schibler U. Resetting of circadian time in peripheral tissues by glucocorticoid signaling. Science. 2000; 289(5488): 2344-7.

31. Simonneaux V, Ribelayga C. Generation of the melatonin endocrine message in mammals: a review of the complex regulation of melatonin synthesis by norepinephrine, peptides, and other pineal transmitters. Pharmacol Rev. 2003; 55(2): 325-95.

32. Larsen PJ. Tracing autonomic innervation of the rat pineal gland using viral transneuronal tracing. Microsc Res Tech. 1999; 46(4-5): 296-304.

33. Teclemariam-Mesbah R, Ter Horst GJ, Postema F, Wortel J, Buijs RM. Anatomical demonstration of the suprachiasmatic nucleus-pineal pathway. J Comp Neurol. 1999; 406(2): $171-82$.

34. Kalsbeek A, Garidou ML, Palm IF, Van Der Vliet J, Simonneaux V, Pevet P, Buijs RM. Melatonin sees the light: blocking GABA-ergic transmission in the paraventricular nucleus induces daytime secretion of melatonin. Eur J Neurosci. 2000; 12(9): 3146-54.

35. Garidou ML, Bartol I, Calgari C, Pevet P, Simonneaux V. In vivo observation of a non-noradrenergic regulation of arylalkylamine $\mathrm{N}$-acetyltransferase gene expression in the rat pineal complex. Neuroscience. 2001; 105(3): 721-9.

36. Perreau-Lenz S, Kalsbeek A, Garidou ML, Wortel J, van der Vliet J, van Heijningen C, Simonneaux V, Pevet P, Buijs RM. Suprachiasmatic control of melatonin synthesis in rats: inhibitory and stimulatory mechanisms. Eur J Neurosci. 2003; 17(2): 221-8.

37. Mendoza J, Drevet K, Pevet P, Challet E. Daily meal timing is not necessary for resetting the main circadian clock by calorie restriction. $J$ Neuroendocrinol. 2008; 20(2): 25160.

38. Feillet CA, Mendoza J, Pevet P, Challet E. Restricted feeding restores rhythmicity in the pineal gland of arrhythmic suprachiasmatic-lesioned rats. Eur J Neurosci. 2008; 28(12): 2451-8.

39. Fernandes PA, Cecon E, Markus RP, Ferreira ZS. Effect of TNF-alpha on the melatonin synthetic pathway in the rat pineal gland: basis for a 'feedback' of the immune response on circadian timing. J Pineal Res. 2006; 41(4): 344-50.

40. Ferreira ZS, Fernandes PA, Duma D, Assreuy J, Avellar MC, Markus RP. Corticosterone modulates noradrenaline-induced melatonin synthesis through inhibition of nuclear factor kappa B. J Pineal Res. 2005; 38(3): 182-8.

41. Saris WH. Effects of energy restriction and exercise on the sympathetic nervous system. Int J Obes Relat Metab Disord. 1995; 19 Suppl 7S17-S23.

42. Mueller PJ. Exercise training and sympathetic nervous system activity: evidence for physical activity dependent neural plasticity. Clin Exp Pharmacol Physiol. 2007; 34(4): 37784.

43. de Vasconcelos AP, Bartol-Munier I, Feillet CA, Gourmelen S, Pevet P, Challet E. Modifications of local cerebral glucose utilization during circadian food-anticipatory activity. Neuroscience. 2006; 139(2): 741-8.

44. Feillet CA, Mendoza J, Albrecht U, Pevet P, Challet E. Forebrain oscillators ticking with different clock hands. Mol Cell Neurosci. 2008; 37(2): 209-21.

45. Yoo SH, Yamazaki S, Lowrey PL, Shimomura K, Ko CH, Buhr ED, Siepka SM, Hong HK, Oh WJ, Yoo OJ, Menaker M, Takahashi JS. PERIOD2::LUCIFERASE real-time 
reporting of circadian dynamics reveals persistent circadian oscillations in mouse peripheral tissues. Proc Natl Acad Sci U S A. 2004; 101(15): 5339-46.

46. Kim MS, Namkoong C, Kim HS, Jang PG, Kim Pak YM, Katakami H, Park JY, Lee KU. Chronic central administration of ghrelin reverses the effects of leptin. Int J Obes Relat Metab Disord. 2004; 28(10): 1264-71.

47. Kalra SP, Bagnasco M, Otukonyong EE, Dube MG, Kalra PS. Rhythmic, reciprocal ghrelin and leptin signaling: new insight in the development of obesity. Regul Pept. 2003; 111(1-3): 1-11.

48. Bodosi B, Gardi J, Hajdu I, Szentirmai E, Obal F, Jr., Krueger JM. Rhythms of ghrelin, leptin, and sleep in rats: effects of the normal diurnal cycle, restricted feeding, and sleep deprivation. Am J Physiol Regul Integr Comp Physiol. 2004; 287(5): R1071-9.

49. Martinez-Merlos MT, Angeles-Castellanos M, Diaz-Munoz M, Aguilar-Roblero R, Mendoza J, Escobar C. Dissociation between adipose tissue signals, behavior and the foodentrained oscillator. J Endocrinol. 2004; 181(1): 53-63.

50. Blum ID, Patterson Z, Khazall R, Lamont EW, Sleeman MW, Horvath TL, Abizaid A. Reduced anticipatory locomotor responses to scheduled meals in ghrelin receptor deficient mice. Neuroscience. 2009.

51. Velkoska E, Morris MJ, Burns P, Weisinger RS. Leptin reduces food intake but does not alter weight regain following food deprivation in the rat. Int $J$ Obes Relat Metab Disord. 2003; 27(1): 48-54.

52. Staszkiewicz J, Horswell R, Argyropoulos G. Chronic consumption of a low-fat diet leads to increased hypothalamic agouti-related protein and reduced leptin. Nutrition. 2007; 23(9): 665-71.

53. de Lecea L, Kilduff TS, Peyron C, Gao X, Foye PE, Danielson PE, Fukuhara C, Battenberg EL, Gautvik VT, Bartlett FS, 2nd, Frankel WN, van den Pol AN, Bloom FE, Gautvik KM, Sutcliffe JG. The hypocretins: hypothalamus-specific peptides with neuroexcitatory activity. Proc Natl Acad Sci U S A. 1998; 95(1): 322-7.

54. Sakurai T, Amemiya A, Ishii M, Matsuzaki I, Chemelli RM, Tanaka H, Williams SC, Richarson JA, Kozlowski GP, Wilson S, Arch JR, Buckingham RE, Haynes AC, Carr SA, Annan RS, McNulty DE, Liu WS, Terrett JA, Elshourbagy NA, Bergsma DJ, Yanagisawa M. Orexins and orexin receptors: a family of hypothalamic neuropeptides and $G$ protein-coupled receptors that regulate feeding behavior. Cell. 1998; 92(5): 1 page following 696.

55. Mignot E, Taheri S, Nishino S. Sleeping with the hypothalamus: emerging therapeutic targets for sleep disorders. Nat Neurosci. 2002; 5 Suppl1071-5.

56. de Lecea L, Sutcliffe JG, Fabre V. Hypocretins/orexins as integrators of physiological information: lessons from mutant animals. Neuropeptides. 2002; 36(2-3): 85-95.

57. Sakurai T. The neural circuit of orexin (hypocretin): maintaining sleep and wakefulness. Nat Rev Neurosci. 2007; 8(3): 171-81.

58. Akiyama M, Yuasa T, Hayasaka N, Horikawa K, Sakurai T, Shibata S. Reduced food anticipatory activity in genetically orexin (hypocretin) neuron-ablated mice. Eur J Neurosci. 2004; 20 (11): 3054-62.

59. Mieda M, Williams SC, Sinton CM, Richardson JA, Sakurai T, Yanagisawa M. Orexin neurons function in an efferent pathway of a food-entrainable circadian oscillator in eliciting food-anticipatory activity and wakefulness. J Neurosci. 2004; 24(46): 10493-501.

60. Niijima A. Neural mechanisms in the control of blood glucose concentration. $J$ Nutr. 1989; 119(6): 833-40.

61. Wasserman DH. Four grams of glucose. Am J Physiol Endocrinol Metab. 2009; 296(1): E11-21.

62. Bansal P, Wang Q. Insulin as a physiological modulator of glucagon secretion. Am J Physiol Endocrinol Metab. 2008; 295(4): E751-61. 
63. La Fleur SE, Kalsbeek A, Wortel J, Buijs RM. A suprachiasmatic nucleus generated rhythm in basal glucose concentrations. J Neuroendocrinol. 1999; 11(8): 643-52.

64. Diaz-Munoz M, Vazquez-Martinez O, Aguilar-Roblero R, Escobar C. Anticipatory changes in liver metabolism and entrainment of insulin, glucagon, and corticosterone in foodrestricted rats. Am J Physiol Regul Integr Comp Physiol. 2000; 279(6): R2048-56.

65. Chiamolera MI, Wondisford FE. Minireview: Thyrotropin-releasing hormone and the thyroid hormone feedback mechanism. Endocrinology. 2009; 150(3): 1091-6.

66. Dayan CM, Panicker V. Novel insights into thyroid hormones from the study of common genetic variation. Nat Rev Endocrinol. 2009; 5(4): 211-8.

67. Hulbert AJ. Thyroid hormones and their effects: a new perspective. Biol Rev Camb Philos Soc. 2000; 75(4): 519-631.

68. Salvador J, Dieguez C, Scanlon MF. The circadian rhythms of thyrotrophin and prolactin secretion. Chronobiol Int. 1988; 5(1): 85-93.

69. Fukuda H, Greer MA, Roberts L, Allen CF, v C, Wilson M. Nyctohemeral and sexrelated variations in plasma thyrotropin, thyroxine and triiodothyronine. Endocrinology. 1975; 97(6): 1424-31.

70. Bernal J, Nunez J. Thyroid hormones and brain development. Eur J Endocrinol. 1995; 133(4): 390-8.

71. Giustina A, Wehrenberg WB. Influence of thyroid hormones on the regulation of growth hormone secretion. Eur J Endocrinol. 1995; 133(6): 646-53.

72. Mariash CN, Oppenheimer JH. Interaction of thyroid hormone and nutritional signals on thyroid hormone action. Mol Cell Endocrinol. 1985; 43(1): 3-13.

73. Ooka H, Segall PE, Timiras PS. Neural and endocrine development after chronic tryptophan deficiency in rats: II. Pituitary-thyroid axis. Mech Ageing Dev. 1978; 7(1): 19-24.

74. Cokelaere M, Decuypere E, Flo G, Darras VM, Kuhn ER. Influence of feeding pattern on thyroid hormones in long-term food-restricted rats. Horm Metab Res. 1996; 28(7): 315-8.

75. Rodriguez M, Rodriguez F, Jolin T. Effect of restricted feeding, fasting, and diabetes on the relationship between thyroid hormone receptor occupancy, growth hormone induction, and inhibition of thyrotropin release in thyroidectomized rats. Endocrinology. 1992; 131(4): 1612-8.

76. Rodriguez M, Rodriguez F, Jolin T, Santisteban P. Comparative effects of food restriction, fasting, diabetes and thyroidectomy on growth hormone and thyrotropin gene expression in the rat pituitary. Eur J Endocrinol. 1995; 133(1): 110-6.

77. Yuwiler A. Effects of steroids on serotonin-N-acetyltransferase activity of pineals in organ culture. J Neurochem. 1989; 52(1): 46-53.

78. Paredes SD, Sanchez S, Parvez H, Rodriguez AB, Barriga C. Altered circadian rhythms of corticosterone, melatonin, and phagocytic activity in response to stress in rats. Neuro Endocrinol Lett. 2007; 28(4): 489-95.

79. Sutcliffe JG, de Lecea L. The hypocretins: excitatory neuromodulatory peptides for multiple homeostatic systems, including sleep and feeding. J Neurosci Res. 2000; 62(2): 1618.

80. Vriend J. Evidence for pineal gland modulation of the neuroendocrine-thyroid axis. Neuroendocrinology. 1983; 36(1): 68-78.

81. Jaworek J, Nawrot-Porabka K, Leja-Szpak A, Bonior J, Szklarczyk J, Kot M, Konturek SJ, Pawlik WW. Melatonin as modulator of pancreatic enzyme secretion and pancreatoprotector. J Physiol Pharmacol. 2007; 58 Suppl 665-80.

82. Dezaki K, Sone H, Yada T. Ghrelin is a physiological regulator of insulin release in pancreatic islets and glucose homeostasis. Pharmacol Ther. 2008; 118(2): 239-49.

83. Davidson AJ, Sellix MT, Daniel J, Yamazaki S, Menaker M, Block GD. Chronic jetlag increases mortality in aged mice. Current Biology. 2006; 16(21): R914-R6. 
84. Salgado-Delgado R, Angeles-Castellanos M, Buijs MR, Escobar C. Internal desynchronization in a model of night-work by forced activity in rats. Neuroscience. 2008; 154(3): 922-31.

85. Mistlberger RE. Circadian Food-Anticipatory Activity - Formal Models and Physiological-Mechanisms. Neuroscience and Biobehavioral Reviews. 1994; 18(2): 171-95.

86. Yerushalmi S, Green RM. Evidence for the adaptive significance of circadian rhythms. Ecology Letters. 2009; 12(9): 970-81.

87. Davidson AJ. Lesion studies targeting food-anticipatory activity. Eur J Neurosci. 2009; 30(9): 1658-64.

88. Challet E, Mendoza J, Dardente H, Pevet P. Neurogenetics of food anticipation. Eur J Neurosci. 2009; 30(9): 1676-87.

89. Burdakov D, Luckman SM, Verkhratsky A. Glucose-sensing neurons of the hypothalamus. Philos Trans R Soc Lond B Biol Sci. 2005; 360(1464): 2227-35.

90. Vollmers C, Gill S, DiTacchio L, Pulivarthy SR, Le HD, Panda S. Time of feeding and the intrinsic circadian clock drive rhythms in hepatic gene expression. Proc Natl Acad Sci U S A. 2009; 106(50): 21453-8.

\section{Figure legends}

\section{Figure 1: Restricted feeding schedules restore a daily rhythm of locomotor activity in}

\section{SCN-lesioned animals.}

Double plotted actogram showing wheel running activity of a rat. Two consecutive days have been represented both on the same line and the same column to improve readability. Wheel revolutions have been recorded and plotted every 5 minutes. The higher the counts, the longer the vertical bar representing a 5 minutes bin. The light/dark cycle is reported on the top of the actogram with white (light) and black (dark) rectangles.

In the first part of the actogram, the rat is rhythmic and mostly active during the night. After bilateral SCN lesion (black arrow), under ad libitum feeding conditions, its locomotor activity becomes arrhythmic. When submitted to a restricted feeding schedule (black rectangle), after a few days, the rat starts to run in the hours preceeding mealtime (Food Anticipatory Activity: FAA ; grey shaded rectangle) thus restoring rhythmic activity.

(Feillet C.A. and Challet E., unpublished data) 


\section{Figure 2: Scheme of secretion profiles of diverse hormones under ad libitum and}

restricted feeding conditions.

Each panel corresponds to one hormone indicated on the left of the graph.

Secretion profiles are predictions derived from various publications (as indicated below) and aligned according to timing of food access: a: Corticosteroids (23-25); b: Melatonin (6, 31, 37) ; c: Leptin (48, 49); d: Ghrelin (48); e: Insulin (64); f: Glucagon (64); g: c-FOS IR in orexin neurons $(58) \mathrm{da}$; h: T4/T3 $(26,68,69,74)$. Plain lines refer to ad libitum feeding conditions while dashed lines show daily variations under RF conditions. The light dark cycle is reported under the graph with white (light) and black (dark) rectangles. For melatonin, calorie restriction was applied in constant darkness, represented by a continuous grey rectangle. Arrows at the bottom of each graph correspond to the beginning of the access to food under RF. 


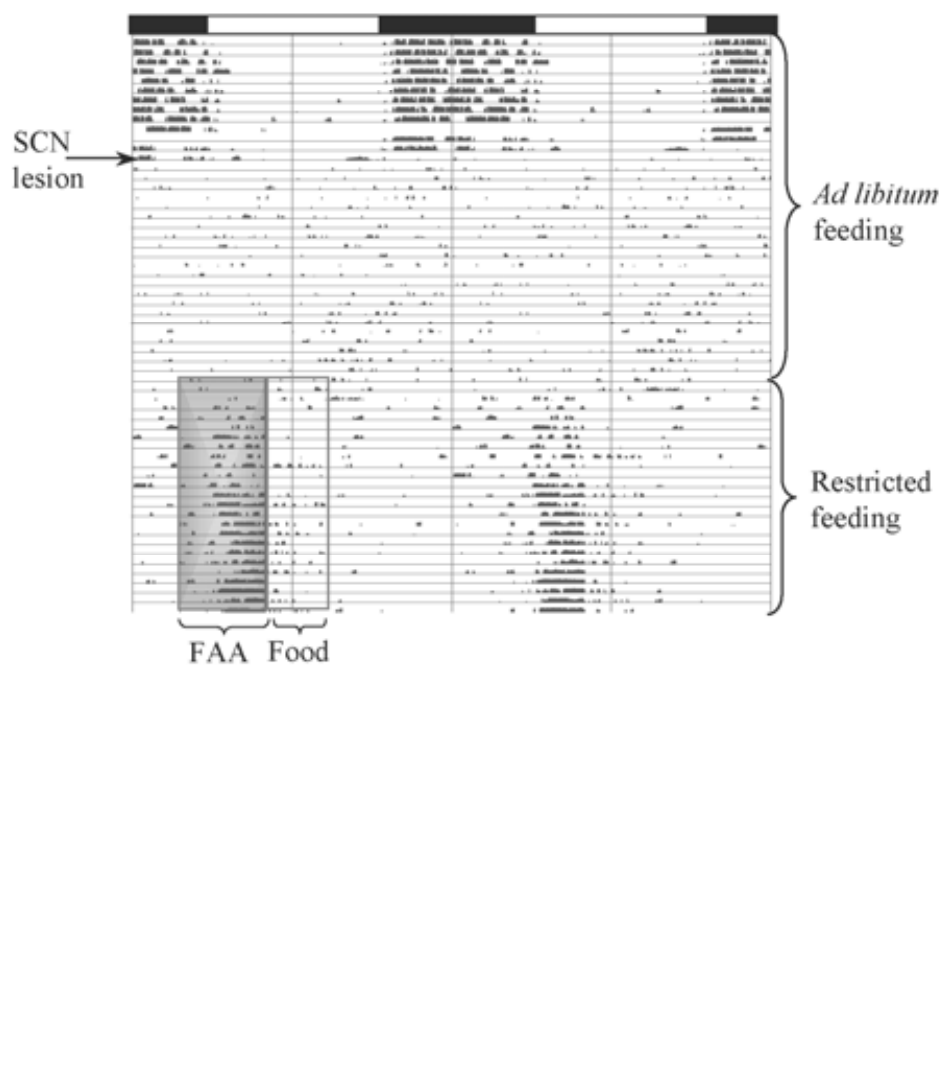

Figure 1 


$\frac{c}{0}$
0
0
0
0
0
0
0
0
0

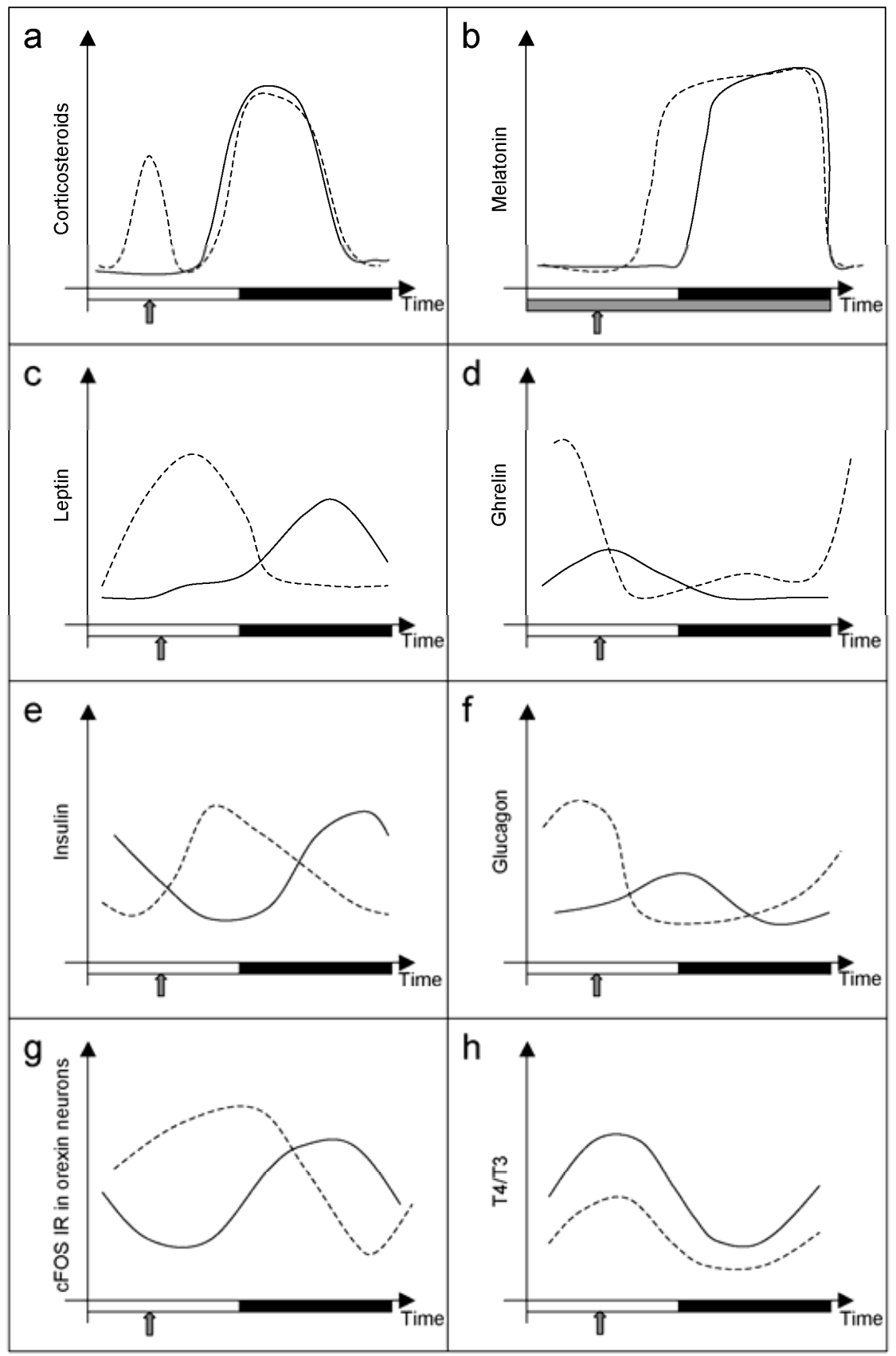

Figure 2 